\title{
Tedious, Tiresome, and Dull: An Unrecognized Problem That We Can Solve
}

\author{
William H. Polonsky \\ Behavioral Diabetes Institute and University of California, San Diego, San Diego, CA
}

\begin{abstract}
Editor's Note: This article is adapted from the virtual address Dr. Polonsky delivered as the recipient of the American Diabetes Association's (ADA's) Outstanding Educator in Diabetes Award for 2020. He delivered the address in June 2020 during the Association's 80th Scientific Sessions, held online as a result of the coronavirus disease 2019.
\end{abstract}

Let's start with the good news. After more than 400 randomized controlled trials and 20 meta-analyses, we can state with some certainty that diabetes self-management education and support (DSMES), especially in group settings, makes a difference. One of the most recent metaanalyses concludes that, compared with control subjects, adults with type 2 diabetes who participate in DSMES programs enjoy significant improvements in AIC, fasting blood glucose, body weight, waist circumference, triglyceride levels, and-perhaps least surprisingly-diabetes knowledge scores (I). To be fair, the authors note that there is marked variability across studies and that many of the documented benefits are often statistically significant, but not necessarily clinically significant. Still, the evidence is consistent and relatively convincing that DSMES programs, especially when done well, can help adults with type 2 diabetes live longer and healthier lives.

But then there's the bad news. The uptake of group DSMES programs continues to be remarkably inadequate. Although the research is limited, it is apparent that many health care professionals (HCPs), and especially those in primary care, are unconvinced that it is worthwhile to refer their patients with type 2 diabetes for DSMES. Because they view it as not particularly beneficial, they do not bother to encourage their patients to seek it out. To be fair, recent surveys suggest that primary care providers (PCPs) may not be as unenthusiastic about DSMES as we suspect. For example, Mehta et al. (2) found that $67 \%$ of PCPs indicated that they regularly refer newly diagnosed adults with type 2 diabetes for DSMES. That sounds impressive, no? But it is important to note that only $2.4 \%$ of the PCPs who were contacted about the survey ever bothered to complete it. Perhaps we should remain a bit skeptical about these findings until we learn more about the attitudes of those remaining $97.6 \%$. In any case, we are in desperate need of more solid research to document and elucidate HCP beliefs and actions regarding DSMES, but the anecdotal evidence points to relatively low HCP enthusiasm.

Of note, regardless of whether it is or isn't due to HCP attitudes and actions, it is well established that newly diagnosed adults with type 2 diabetes rarely receive any form of DSMES. In the United States, among those who had private insurance, only $6.8 \%$ of those who had been diagnosed within the past 12 months were found to have an insurance claim for DSMES in 20II-20I2 (3). Recent data from the United Kingdom indicate that $<30 \%$ of adults with type 2 diabetes who are referred to a DSMES program ever go (4). Even worse, for that small fraction of individuals who are referred and do begin a multi-session DSMES program, the chances are that most of them will never complete it. In the American Association of Diabetes Educator's 20I8 National Practice Survey (5), when diabetes educators were asked to estimate how many of the participants in their DSMES group programs completed at least $75 \%$ of the course, the mean result was $27.3 \%$. Please note that this is merely an estimate from that minority of educators who responded to the survey. Could the actual numbers be even worse?

It is striking how little solid data we have on DSMES program retention in the real world. To be fair, Chrvala et al. 
(6) observed that DSMES retention rates are relatively high in well-designed study settings, but this is unlikely to be reflective of outcomes in real-world settings. When I have the opportunity to question my CDCES colleagues around the United States, there is widespread agreement that such data should be collected and reported, and there is also significant discomfort because most quietly acknowledge that retention in their own programs is disappointing. Perhaps it is this sense of disquiet and embarrassment, at least partially, that contributes to the lack of active research in this area.

Still, some programs have undertaken such a brave reckoning. Cunningham et al. (7) examined the outcomes from their group medical visit program that involved four onceweekly meetings. At each of the 2-hour sessions, the first hour was devoted to a family medicine resident, while the second hour was a presentation from a diabetes educator. Among the 230 adults with type 2 diabetes who had attended at least the first session, the study investigators found that the median number of total visits attended was one. In other words, the majority of patients never returned after that first session. Even worse, only $3.9 \%$, or 9 of 230 participants, attended all four of the once-weekly meetings. Could these grim results be representative of DSMES group retention across the United States? It is possible, but we don't know.

\section{What Might Explain These Findings?}

In total, a large body of data points to the potential value of DSMES group programs, but it is apparent few adults with type 2 diabetes are ever referred to them, and when referred, relatively few actually go. Of those who do attend, our limited evidence to date suggests that most of them drop out before the program ends. So, what is the problem? Why is retention in DSMES programs so low? We aren't certain, but it strikes me that educators are often telling themselves this comforting story: "We educators are like retailers, and we run this beautiful store. Our store is well-appointed and is chockful of beautiful products (i.e., our DSMES programs). We are so proud of our store, even though we don't seem to have many customers (especially not repeat customers). Still, if customers (i.e., people with diabetes) never come to our store, or if they come once and never come back, then something obviously is wrong with them. They don't appreciate how wonderful our store is. It is their fault for being ignorant and unappreciative."

There is a modicum of research in support of this tale. Gucciardi et al. (8) interviewed 97 individuals who had dropped out of their DSMES program and found that participants' work schedules and the center's limited hours of operation were the most frequently cited reasons for attrition. Apparently, for this group, the critical problem was that attending the program had become inconvenient. If adults with type 2 diabetes decide to neglect the potential health benefits of DSMES merely because of "inconvenience," then perhaps it is their fault, no? One of the basic (and well-documented) tenets of behavioral economics is that convenience (or lack of convenience) influences behavior. If DSMES program locations were more attractive and easier to reach, and if the programs were offered during times when people find it easier to attend, then retention might improve. But consider once again our retail shop analogy. Would moving our DSMES "shop" closer to our customers and perhaps expanding our store hours solve the problem of our empty store? Perhaps. But that improvement, I would suggest, is likely to be minimal.

Lack of convenience is less likely to deter a positive action (say, attending a DSMES program) when that positive action is considered to be truly worthwhile. Consider how the constraints of your own impossibly busy work schedule might be something you would work hard to overcome if you viewed the potential action as truly crucial to your health. If you felt it was vitally important, you would be much more likely to make the time. And so, when the DSMES dropout communicates that "Gee, sorry, but I'm just too busy to continue this program," perhaps what he or she is really thinking and feeling underneath that statement is-at least to some degree-"Your program really isn't that worthwhile. If I had found it more engaging, meaningful, and relevant to my life, I would have definitely come back."

These terrible retention rates indicate that our DSMES customers are voting with their feet, and we need to courageously look at why this might be so. Problematic attrition in our programs isn't because our patients are dumb or because our programs are inconvenient (although the latter certainly has a role to play, especially when poverty, job constraints, transportation hassles, or other social determinants of health loom large), but the bigger problem is more likely because of us: our programs are often flat-out boring. We must face the fact that we are often the problem, not our patients.

\section{A Closer Look at Boredom}

After decades of research investigating the phenomenon of boredom (9), scientists have become highly skilled at inducing boredom in the laboratory setting. Examples include having participants watch a terribly long video of 
TABLE 1 How Would Your Education Program Participants Respond?

\begin{tabular}{ll}
\hline Time seemed to pass by slower than usual. & $\square$ Agree \\
\hline I felt stuck in a situation that seemed irrelevant. & $\square$ Agree \\
\hline It all seemed repetitive and routine to me. & $\square$ Agree \\
\hline I felt bored. & $\square$ Agree \\
\hline I felt forced to think about things that had no real value to me. & $\square$ Agree \\
\hline I wished I was doing something more exciting. & $\square$ Agree \\
\hline
\end{tabular}

people hanging laundry, asking participants to proofread address labels for extended periods, and-my personal favorite-asking people to view a conversation of a man quietly explaining his job at an office supply company, describing a conversation with a client, eating lunch at his desk, and explaining the determinants of cardstock prices (Io). Furthermore, thanks to these pioneering researchers, we now also have effective means for assessing boredom. One well-known measure is the Multidimensional State Boredom Scale (MSBS) (II). A subset of MSBS items is shown in Table I; please consider how your own DSMES participants might respond to these items at the conclusion of your program. As uncomfortable as it might be to consider this, perhaps our DSMES programs have unintentionally become effective boredom-induction strategies. In that case, it is no wonder that attrition rates are so high.

To be fair, almost all of the educators whom I've had the chance to meet over the past 30 years-thousands of them from around the world-are pleasant, charming, and knowledgeable. They're respectful of their DSMES program participants, they care about them, and they want to make a difference. In most situations, they are not boring people. And yet, our patients are dropping out often because-as I am arguing - they just don't find these programs meaningful, engaging, and worthwhile. They are bored.

Of course, this is not always the case, but it is more likely to be true in programs where the certified diabetes care and education specialist (CDCES) staff are mostly lecturing, where there is minimal effort to encourage questions from and interaction with participants, where the CDCES staff does not clarify why all of this information is personally meaningful for the people in the room, and where staff may encourage behavior change, but don't effectively help participants in a personalized manner figure out how to implement those changes in their already complicated lives.
I have never met a CDCES who aspired to be boring. It happens, though, when educators feel pressured by their health care systems to deliver unreasonable quantities of information in too short a period of time. It happens when educators become fearful that too much interaction with program participants will make it impossible to deliver all of the information they feel obligated to impart. Additionally, when educators are concerned that they will not be able to answer participants' difficult questions, it becomes too easy to fall back into the lecture format and then discourage participants from asking questions.

\section{Theory $\neq$ Practice}

None of this really seems to make any sense, though. Most knowledgeable educators recognize that DSMES has changed over the years, and that — thanks to good research — the field has moved away from a focus on lecturing and no longer conceptualizes DSMES participants as passive recipients of information. DSMES in the 2Ist century, as described in a thorough review by Hermanns et al. (I2), places:

“... an emphasis on informed decision-making, self-care behaviour, problem-solving, and active collaboration and participation with the health care team to improve not only clinical outcomes but also health status, coping with the chronic condition, and quality of life."

This sounds terrific, but does it reflect current DSMES practice? Hermanns et al. (I2) draw the reader back to reality with this sobering (and very tactful) conclusion:

“... it remains unclear to what extent the theoretical concept of the examined DSME programmes are actually realized and the conduct of the programme was in line with its curriculum. A specific challenge here is that the claim to personalize DSME to the individual needs and problems can challenge the curricular conduct of the DSME programme." 
In other words, our 2Ist century concepts of what should be occurring in DSMES may not match what is actually happening in practice. There is pressure to deliver more and more information in the group setting, while at the same time wishing to personalize it for each program participant. These two goals are in conflict, with the former often outweighing the latter. The result? Boredom for the participant, leading to disengagement and embarrassing rates of program attrition.

\section{It Doesn't Have to Be This Way}

Overcoming DSMES boredom means remembering what scientific research has already taught us. We know why and how people learn and change and why they find interactions engaging and worthwhile. Not surprisingly, it's not typically from lectures, lectures, and more lectures. It's through conversation, stories, and personal discovery. DSMES needs to be restructured to better support that style of learning.

\section{Solution 1: Cut Down on the Content}

If there is to be time for conversation, stories, and personal discovery, then we must reduce the content that has been squeezed into DSMES programs. It is too much! For many of my educator colleagues, this is painful to consider, but it is essential. And the DSMES standards do not require all that is being typically delivered (13). Here are a few suggestions for cutting back on content:

- Addressing participants' concerns about their own medications is more important than explaining how each class of antidiabetic medications works. In many DSMES programs, much time is spent detailing how each class of oral and injectable antidiabetic medication works, with little recognition of the fact that participants may not care, may only be interested in what they are currently taking, and/or are deeply skeptical and suspicious about what they have been prescribed.

- This will seem like a sacrilege to many of our CDCES colleagues, but the ability to estimate carbohydrates and read food labels, which is a time-consuming focus of many DSMES programs, may be less important than helping to convince DSMES group participants that making positive dietary changes may be personally worthwhile; to identify a single, practical change in their diet that they may feel willing and able to make; and to support participants with developing a personal plan for how they might succeed with such a change.

- The vast majority of DSMES participants are not interested in lengthy biochemical explanations of how long-term complications develop or how hemoglobin becomes glycosylated. They just aren't. Instead, consider that participants are likely to be interested in understanding their own $\mathrm{AIC}$ value and why that number might be important for their own health.

\section{Solution 2: Make Programs Personally Engaging and Meaningful}

In every DSMES session, there must be time to discuss, review, and reinforce the "why bothers" - the reasons why every participant is attending this program. In our programs at the Behavioral Diabetes Institute, this starts by reminding participants about the well-established good news about diabetes: "With good care and effort, the odds are pretty good that you can live a long healthy life with diabetes." But our participants (and yours) may not so easily believe this. This needs to be part of an ongoing conversation with all participants - to understand how they think about the long-term risks of diabetes as well as the potential benefits of taking positive action, and to encourage them to believe that their own actions (including their participation in the DSMES program) will be worthwhile.

It is also crucial to keep a clear-eyed focus on participants' questions and concerns. Although time may be limited during a presentation and it may not be possible to answer all questions, DSMES staff can honor their participants by having a white board or flip chart at hand where they can list all questions and concerns as they arise (even though they may not be able to respond fully until much later). In this manner, the program is viewed as more personally relevant by each participant, leading to ongoing interest and engagement.

Finally, at the end of each DSMES session, why not conclude by asking participants about their impressions of the session? What worries them about what they've heard? What do they believe about what they've heard? What did they find valuable and not so valuable? What might they now try or do differently? Because time is limited, it may not be possible for all participants to respond, but this process encourages a sense of personal ownership of the program. This DSMES program is for them; it is their program.

\section{Solution 3: Remember the First Tenet of Alcoholics Anonymous}

We must stop looking away and admit that we, as DSMES program staff, just might have a problem. As a first step, keep careful track of the no-show and dropout rates in your programs. Is there a significant problem, or not? Then locate and interview the dropouts, asking them about what was missing for them. If they respond, "Well, I got busy" or "It was too far away," or "It was inconvenient," dive deeper. 
Without any judgment, inquire more broadly about their DSME program experiences: "What was the program really like for you? What were the pluses and minuses?" "What would have made the program more valuable for you?" As noted above, there is remarkably little available data on this topic. And so, regardless of the results, please be courageous, like Cunningham et al. (7), and seek to publish those findings. If we are to solve the problem, we must first recognize and acknowledge its scope.

As a result, we might expect that DSMES program directors will become more enthused about revising and reinventing their programs. Imagine what could be accomplished with a DSMES program that was unleashed by the powers of our own creativity and innovation. Imagine a DSMES program that ignited participants' interest in caring for their diabetes. Imagine a DSMES program that wasn't boring.

\section{Just Say No to Boredom}

The bottom line is this: Educators must refuse to be boring. There is no longer any need to be straitjacketed by the belief that we must lecture, lecture, and lecture. DSMES programs must be more than just a process of shoveling facts onto participants. The desperate need for DSMES programs to become more engaging and meaningful is not a new idea. Among others, pioneering efforts from Anderson and Funnell (I4) and Brackenridge and Swenson (I5) have led the way toward the development of innovative programs that make a difference. Let's follow their lead and focus our efforts on the transformation of DSMES by remembering the key principle of effective education. To paraphrase Plutarch (I6), "Education is not the filling of a pail, but the lighting of a fire."

\section{DUALITY OF INTEREST}

No potential conflicts of interest relevant to this article were reported.

\section{REFERENCES}

1. Odgers-Jewell K, Ball LE, Kelly JT, Isenring EA, Reidlinger DP, Thomas R. Effectiveness of group-based self-management education for individuals with type 2 diabetes: a systematic review with meta-analyses and meta-regression. Diabet Med 2017;34: 1027-1039

2. Mehta S, Mocarski M, Wisniewski T, Gillespie K, Narayan KMV, Lang K. Primary care physicians' utilization of type 2 diabetes screening guidelines and referrals to behavioral interventions: a survey-linked retrospective study. BMJ Open Diabetes Res Care 2017;5:e000406

3. Li R, Shrestha SS, Lipman R, Burrows NR, Kolb LE, Rutledge S; Centers for Disease Control and Prevention (CDC). Diabetes self-management education and training among privately insured persons with newly diagnosed diabetes: United States, 2011-2012. MMWR Morb Mortal Wkly Rep 2014;63:1045-1049

4. National Health Service. National Diabetes Audit, 2016-17: Report 1: Care Processes and Treatment Targets. Leeds, U.K., National Health Service Digital, 2018

5. Rinker J, Dickinson JK, Litchman ML, et al. The 2017 diabetes educator and diabetes self-management education national practice survey. Diabetes Educ 2018;44:260-268

6. Chrvala CA, Sherr D, Lipman RD. Diabetes self-management education for adults with type 2 diabetes mellitus: a systematic review of the effect on glycemic control. Patient Educ Couns 2016;99: 926-943.

7. Cunningham AT, Delgado DJ, Jackson JD, et al. Evaluation of an ongoing diabetes group medical visit in a family medicine practice. J Am Board Fam Med 2018;31:279-281

8. Gucciardi E, Demelo M, Offenheim A, Stewart DE. Factors contributing to attrition behavior in diabetes self-management programs: a mixed method approach. BMC Health Serv Res 2008;8:33

9. Koerth-Baker M. Boredom gets interesting. Nature 2016;529: 146-148

10. Markey A, Chin A, Vanepps EM, Loewenstein G. Identifying a reliable boredom induction. Percept Mot Skills 2014;119:237-253

11. FahIman SA, Mercer-Lynn KB, Flora DB, Eastwood JD. Development and validation of the multidimensional state boredom scale. Assessment 2013;20:68-85

12. Hermanns N, Ehrmann D, Finke-Groene K, Kulzer B. Trends in diabetes self-management education: where are we coming from and where are we going? A narrative review. Diabet Med 2020;37: 436-447

13. Powers MA, Bardsley JK, Cypress M, et al. Diabetes selfmanagement education and support in adults with type 2 diabetes: a consensus report of the American Diabetes Association, the Association of Diabetes Care \& Education Specialists, the Academy of Nutrition and Dietetics, the American Academy of Family Physicians, the American Academy of PAs, the American Association of Nurse Practitioners, and the American Pharmacists Association. Diabetes Care 2020;43:1636-1649

14. Anderson RM, Funnell MM. The art and science of diabetes education: a culture out of balance. Diabetes Educ 2008;34: 109-117

15. Brackenridge B, Swenson K. Discovering diabetes: achieving target blood glucose control through a behavioural approach to insulin and food self-management. Br J Diabetes Vasc Dis 2004;4: $117-120$

16. Quote Investigator. The mind is not a vessel that needs filling, but wood that needs igniting: William Butler Yeats? Plutarch? Socrates? Plato? Apocryphal? Available from https:// quoteinvestigator.com/2013/03/28/mind-fire. Accessed 26 June 2020 